AN. MED. INTERNA (Madrid) Vol. 18, N. $^{\circ} 9$, pp. $481-482,2001$

\title{
Intoxicación fatal por Lepiota bruneoincarnata
}

\author{
A. PUIG HERNÁNDEZ, C. CHUMILLAS CÓRDOBA, J. CAMPRODÓN CALVERAS, \\ E. DE FRANCISCO ENCISO*, M. ${ }^{a}$ P. FURIÓ MARCO, G. FERRÁN MARTÍNEZ \\ Servicio de Urgencias. Hospital General. Castellón. *Institut Valencia d'Estudis en Salut \\ Pública. Castellón
}

\author{
LEPIOTA BRUNEOINCARNATA FATAL INTOXICATION
}

\section{RESUMEN}

La intoxicación por setas no suele ser habitual, pero es de elevada incidencia en el periodo otoñal y la gravedad de los síntomas, en algunos casos, justifica la revisión del tema, a propósito de un caso familiar acaecido recientemente en nuestro medio.

En este artículo presentamos dos casos de intoxicación por Lepiota bruneoincarnata en dos pacientes de la misma familia, que desarrollaron afectación visceral con alteración de la función hepática, con desenlace fatal en uno de ellos.

La rareza, gravedad de los síntomas y su semejanza con las intoxicaciones por Amanita phalloides nos ha parecido interesante para su publicación.

PALABRAS CLAVE: Intoxicación por setas. Lepiota. Lepiota brune oincarnata.

\begin{abstract}
Mushroom poisoning does not occur very often but in autumn the incidence increases. The seriousness of symptoms in some cases justif;es the revision of this issue just the event of one family cases ocurred recently in our environment. In this paper we are presenting two cases of intoxication by Lepiota bruneoincarnata in two patients of the same family who suffers visceral involvement with a hepatic strike down and death in one of the patients. The rarity, and seriousness of symptoms together with the similarity with intoxication by Amanita phalloides seem very interesting to us to be published.
\end{abstract}

KEY WORDS: Mushroom-poisoning. Lepiota. Lepiota bruneoincar nata.

Puig Hernández A, Chumillas Córdoba C, Camprodón Calveras J, de Francisco Enciso E, Furió Marco MP, Ferrán Martínez G. Intoxicación fatal por Lepiota bruneoincarnata. An Med Interna (Madrid) 2001; 18: 481-482.

\section{INTRODUCCIÓN}

La intoxicación por setas venenosas aunque infrecuente puede tener graves consecuencias para la salud. En todos los ámbitos es bien conocida la toxicidad de la Amanita phalloi des, pero existen otras setas, entre ellas las lepiotas, menos conocidas incluso en el medio sanitario, con una toxicidad similar a la anterior (1).

El objetivo de este artículo es destacar la gran tradición de consumo y recolección de setas en nuestro país, desconociendo en muchos casos la naturaleza de la seta que se recoge confundiéndola con especies comestibles, las lepiotas tienen características morfológicas que facilitan el error en micólogos neófitos o poco atentos (2).

Dentro de los síndromes de intoxicación tardía y que constituyen los de peor pronóstico se encuentra el caso que nos ocupa. La seta tóxica fue recogida en un parque urbano, en las inmediaciones de la ciudad de Castellón, al parecer el pacien- te había recolectado con anterioridad setas comestibles de aspecto casi idéntico. Posteriormente se recogieron setas en la misma zona, que fueron reconocidas por el paciente como las ingeridas e identificadas por un micólogo como Lepiota bru neoincarnata.

\section{CASO APORTADO}

Varón de 61 años, que tras 10 horas de haber ingerido setas, identificadas con posterioridad como pertenecientes al género Lepio $t a$, presenta cuadro de vómitos y diarrea. Su hija, de 29 años, presenta clínica idéntica con igual antecedente, si bien se refiere una ingesta en mayor cuantía por parte del padre. Al ingreso en el Servicio de Urgencias, el varón presentaba regular estado general, con constantes hemodinámicas estables, tensión arterial 140/80 mmHg, temperatura $36,2{ }^{\circ} \mathrm{C}$, auscultación cardio-pulmonar normal y palpación abdominal ligeramente dolorosa, sin peritonismo. En las pruebas complementarias hallamos hemograma normal, el estudio de coagu-

Trabajo aceptado: 30 de diciembre de 1999

Correspondencia: A. Puig Hernández. Paseo Monte Picayo, n 13, Urbanización Los Monasterios. 46530 Puzol. Valencia. 
lación mostró índice de Quick del 82\%, la glucemia fue de $139 \mathrm{mg} / \mathrm{dl}$ y la creatinina en sangre de $1,21 \mathrm{mg} / \mathrm{dl}$. La mujer al ingreso mostraba buen estado general, con exploración clínica normal y resultados analíticos dentro del rango de la normalidad. Tras doce horas de ingreso en la Unidad de Corta Estancia, el varón sufre deterioro clínico con hipotensión, presión venosa central de $5 \mathrm{~cm} \mathrm{H}_{2} \mathrm{O}$, acidosis metabólica con gasometría arterial: $\mathrm{pH} 7,20, \mathrm{PO}_{2} 97 \mathrm{mmHg}, \mathrm{pCO}_{2} 9$ $\mathrm{mmHg}$, bicarbonato actual $8 \mathrm{mEq} / \mathrm{l}$, hipoglucemia de $52 \mathrm{mg} / \mathrm{dl}$, creatinina de $3,3 \mathrm{mg} / \mathrm{dl}$ y alteración de la función hepática con cifras de GOT 2804 U/l, GPT 2318 U/l, I Quick 28\%, TTPA 52" (control 36 "), leucocitosis de $20.000 \mathrm{~mm}^{3}$ y hematocrito $52 \%$.

Ante la evolución del cuadro se traslada al paciente a la Unidad de Cuidados Intensivos, donde se confirma la colocación de la sonda nasogástrica a nivel duodenal a efecto de practicar aspiración continua y administrar carbón activado, se instaura pauta de penicilina $\mathrm{G}$ sódica a altas dosis 5 millones $/ 6 \mathrm{~h}$, silimarina $350 \mathrm{mg} / \mathrm{h} / 6 \mathrm{~h}$ perfusión de líquidos y aporte de bicarbonato.

Una vez estabilizado el paciente se deriva a hospital de referencia con indicación de trasplante ante fallo hepático agudo (3).

El paciente evolucionó desfavorablemente falleciendo horas después. La paciente precisó de ingreso en la unidad de cuidados intensivos, con alteraciones en la hemostasia: I. Quick 55\%, citolisis hepática: GOT 109 U/l, GPT 95 U/l; gasometría arterial con acidosis metabólica compensada: $\mathrm{pH} 7,37, \mathrm{PO}_{2} 99 \mathrm{mmHg}, \mathrm{PCO}_{2} 34 \mathrm{mmHg}$, exceso de bases: -4 , bicarbonato $19 \mathrm{mEq} / \mathrm{l}$, ácido láctico $3,5 \mathrm{mmol} / \mathrm{l}$. Bioquímica y hemograma sin alteraciones. Durante la estancia en UCI y tratada con fluidos, penicilina $\mathrm{G}$ sódica, tratamiento antiabsorción y vitamina K evolucionó favorablemente pasando a la unidad de medicina interna a las 72 horas.

\section{DISCUSIÓN}

La intoxicación por Lepiota bruneoincarnata es poco frecuente pero de elevada mortalidad. Se incluye dentro de los síndromes de aparición tardía, junto con la Amanita Faloides, según la clasificación descrita en los protocolos de la Sociedad Española de Toxicología (4), de gran utilidad en la práctica clínica, que distingue entre:

1. Síndromes de aparición precoz: en general benignos.

-Inocybes y clitocybes $\rightarrow$ síndrome colinérgico.

Latencia: 1,5-2 horas. En la fase de estado aparecen alteraciones digestivas con: náuseas, vómitos, diarrea y dolor abdo- minal, bradicardia, hipotensión, síncope, miosis y temblores. Evolución favorable en pocas horas. Tratamiento: respetar vómitos, rehidratar y atropina.

- Amanita pantepina y muscaria $\rightarrow$ síndrome atropínico.

Latencia: 1-4 horas. La fase de estado cursa con: náuseas, vómitos, midriasis, taquicardia, cuadro seudoebrioso y delirio atropínico. Evoluciona hacia la curación espontánea en 24 horas. Tratamiento: respetar los vómitos, antiespasmódico no atropínico y diacepam.

-Entoloma lividum, lactaria, tricoloma $\rightarrow$ síndrome disentérico.

Latencia 2-3 horas. La fase de estado cursa con vómitos y dolor abdominal. Evoluciona a la curación espontánea.

2 . Síndromes de aparición tardía. Peor pronóstico.

-Amanita faloides, verna o virosa, lepiota bruneoincar nata.

Latencia 7-48 horas. La fase de estado se inicia con un cuadro de gastroenteritis aguda: nauseas, vómitos, diarrea coleriforme, cefaleas, calambres musculares y dolor abdominal la duración suele ser de 2 a 3 días y evoluciona a la curación o muerte por shock hipovolémico. Posteriormente aparecen las lesiones viscerales: citolisis hepatocitaria, ictericia y coma, en la última fase se instaura la insuficiencia renal secundaria. Tratamiento: Se coloca sonda nasogástrica de aspiración contínua (ciclo enterohepático), carbón activado y purgantes. Rehidratación con suero fisiológico. Penicilina G-sódica 300.000 U/kg/día fraccionando las dosis de 1 a $4 \mathrm{~h}$. Diuresis forzada neutra. Plasmaféresis o hemoperfusión en las 48 primeras horas. Pronóstico: Entre el $3^{\circ}$ y $4^{\circ}$ día la mortalidad es del $20-25 \%$.

- Giromitra esculenta.

Latencia: 10-18 horas. Fase de estado: gastroenteritis banal, alteraciones neurológicas, citolisis hepática y hemolisis. Tratamiento sintomático. La mortalidad es del 2 al $8 \%$.

-Cortinarius orelanius.

Latencia: 10-15 días. Fase de estado: comienzo insidioso, con náuseas y dolor abdominal apareciendo posteriormente nefropatía tubulointersticial y necrosis hepática. El tratamiento es sintomático. Pronóstico: evoluciona hacia la curación o la insuficiencia renal crónica.

\section{Bibliografía}

1. Ramírez P, Parrilla P, Sánchez F et al. Fulminant hepatic failure after Lepiota mushroom poisoning. J Hepatol 1993; 19: 51-54.

2. Soyez C, Jonville-Bera AP, Crece J et al. Intoxication grave par Lepiota jossrandii Arch Pediatr 1994; 1: 214.
3. Skaare VK. Mushroom poisoning: an indication for liver trasplantation. J Transpl Coord 1997; 7(3): 141-3

4. Protocolos de Intoxicaciones. Sociedad Española de Toxicología. 